SRC TR $\quad 90-41$

A Note on the Comparison between Bernoulli and Limited Policies in Vacation Models

By

T. Tedijanto 


\title{
A NOTE ON THE COMPARISON BETWEEN BERNOULLI AND LIMITED POLICIES IN VACATION MODELS
}

\author{
Tedijanto* \\ Electrical Engineering Department and Systems Research Center ${ }^{\dagger}$ \\ University of Maryland, College Park, Maryland 20742
}

January 1990

\begin{abstract}
A vacation model is a single-server queueing system where the server occasionally takes a vacation, i.e., becomes unavailable to the customers for a period of time. Under a limited service policy with parameter $m, m=1,2, \ldots$, the server can serve a maximum of $m$ consecutive customers before it has to take a vacation. Under a Bernoulli service policy with parameter $p, 0 \leq p \leq 1$, at every service completion, the server serves the next customer (if available) with probability $p$ or starts a vacation with probability $1-p$. Under both policies, the server takes a vacation if no customer is present. In this note, the waiting time under a Bernoulli policy with parameter $p$ is shown to dominate that under a limited policy with parameter $m$ when the parameters are related by $p=(m-1) / m$ and the vacation lengths are identical and deterministic. The comparison is in the convex increasing ordering and holds both in the transient and steady-state regimes.
\end{abstract}

Keywords: Queueing Systems, Vacation Models, Stochastic Comparison.

- This work was supported partially through NSF Grant ECS-83-51836 and partially through a grant from AT\&T Bell Laboratories.

With the support of the National Science Foundation Engineering Research Centers Program NSFD CDR-85-00108. 


\section{Introduction}

A vacation model is a $G I / G I / 1$ queue where under some prespecified conditions, the server takes a vacation, i.e., becomes unavailable to the customers for a period of time. Such a model arises naturally in a wide variety of computer and communications systems. In these systems, vacations typically correspond to secondary tasks or routine maintenance periods that the processor has to perform or undergo from time to time. Vacation models have also been used to study other queueing models, in particular polling systems. For a complete survey on vacation models and their applications, the reader is referred to Doshi [3].

In a vacation model, the conditions under which the server takes a vacation are determined by the service policy. In this note, we consider two nonexhaustive service policies, the so-called limited and Bernoulli policies. Fuhrmann [4] obtained an upper bound for the steady-state mean waiting time of a symmetric polling system under a limited service policy. Servi and Yao [8] subsequently showed that, in the context of vacation models, this bound was exactly the mean waiting time under a Bernoulli policy (with suitably chosen parameters). Servi and Yao also obtained other comparison results which suggest that the steady-state waiting times for limited and Bernoulli policies can be compared in the increasing convex (icx) ordering. In this note, we show that such a comparison indeed holds when the vacation periods are identical and deterministic. In fact, the comparison holds not only in the steady-state but also in the transient regime.

In the next section, we present a precise description of vacation models together with the notation used throughout the note. In Section 3, we introduce the notion of icx ordering. There, we also note some preliminary results crucial to the subsequent development. In Section 4 and 5 , we derive the comparison result for the transient and steady-state cases, respectively.

\section{The Model and Notation}

In this section, we give a precise description of a vacation model under the exhaustive, limited and Bernoulli service policies. First, we introduce the notation used throughout the note.

The following random variables $(n=1,2, \ldots)$ constitute a vacation model:

$\tau_{n}=$ the time between the $(n-1)$ st and the nth arriving customer;

$\sigma_{n}=$ the length of the $n$th service;

$r_{n}=$ the length of the $n$th vacation period.

We assume that the sequences $\left\{\tau_{n} . n=1,2, \ldots\right\},\left\{\sigma_{n}, n=1.2, \ldots\right\}$ and $\left\{V_{n}, n=1,2 \ldots\right\}$ are mutually independent and that each sequence is composed of i.i.d. random rariables with a general common distribution.

We define the following $(n=1,2, \ldots)$ :

$C_{n}=$ the $n$th arriving customer;

$A_{n i}=$ the arrival time of $C_{n}\left(=\sum_{j=1}^{n} \tau_{j}\right)$;

$D_{n}=$ the departure (i.e., service completion) time of $C_{n}$;

$r_{n}=$ the number of vacations completed up to the service completion of $C_{n}$ :

$q_{n}=$ the number of customers in the system left behind by $C_{n}$ :

$s_{n}=$ the number of customers up to and including $\left({ }_{n}\right.$ that have been served since the end of the $r_{n}$ th racation;

$H_{n}=D_{n}-\sigma_{n}-A_{n}=$ the waiting time of $C_{n}$. 
A vacation model under the exhaustive service policy is described as follows. At time $D_{n}$, $n=1,2, \ldots$, the server finishes serving $C_{n}$. If $q_{n}>0$ (i.e., $C_{n+1}$ is waiting in the queue), the server immediately serves $C_{n+1}$ (thus customers are served on a FCFS basis). If $q_{n}=0$, the server takes a vacation and keeps on taking them (of lengths $V_{r_{n}+1}, V_{r_{n}+2}, \ldots$ ) until at least one customer is present when it returns from a vacation. Doshi [3] refers to a vacation model in which the server behaves in this manner if $q_{n}=0$ as a multi-vacation model.

Let us denote a limited service policy with parameter $m, m=1,2, \ldots$, by $L(m)$ and a Bernoulli service policy with parameter $p, 0 \leq p \leq 1$, by $B(p)$. In the case $q_{n}=0$, the behavior of the server under the policies $L(m)$ and $B(p)$ is similar to that under the exhaustive policy. If $q_{n}>0$ under $L(m)$, the server's action is determined by $s_{n}$ : If $1 \leq s_{n}<m$, the server serves $C_{n+1}$; otherwise (i.e., if $s_{n}=m$ ), it takes one vacation (of length $V_{r_{n}+1}$ ) and then serves $C_{n+1}$. Under the policy $B(p)$, the server's action for $q_{n}>0$ is determined by the random variable $x_{n}$ : If $x_{n}=0$, the server serves $C_{n+1}$; if $x_{n}=1$, it takes one vacation and then serves $C_{n+1}$. The sequence $\left\{x_{n}, n=1,2, \ldots\right\}$ is composed of i.i.d. $\{0,1\}$-valued random variables with

$$
P\left[x_{n}=1\right]=1-p \quad n=1,2, \ldots
$$

and is assumed to be independent of the random variables $\left\{\tau_{n}, \sigma_{n}, V_{n}, n=1,2, \ldots\right\}$.

In the sequel, we shall use superscript $B(p)$ (resp. $L(m)$ ) for any quantity associated with the service policy $B(p)$ (resp. $L(m)$ ). We also make the following assumption (A), where

(A) At time $t=0$, the system is empty and the server starts a vacation at $t=0$.

\section{Preliminaries}

We note several simple relations which are crucial to the discussion of the next sections. First, observe that the server in a vacation model is always doing one of two things, either serving a customer or taking a vacation. Therefore, for $i=B(p), L(m)$ we have that

$$
D_{n}^{i}=\sum_{j=1}^{n} \sigma_{j}+\sum_{k=1}^{r_{n}^{i}} V_{k}, \quad n=1,2, \ldots
$$

From the definitions of $B(p)$ and $L(m)$ policies in the previous section, we can write the following recursive equations describing the crolution of $r_{n}$. Under the service policy $B(p)$, we have

$$
r_{n+1}^{B(p)}= \begin{cases}r_{n}^{B(p)}+x_{n} & \text { if } q_{n}^{B(p)}>0 \\ \min \left\{l>r_{n}^{B(p)}: \sum_{j=1}^{n} \sigma_{j}+\sum_{k=1}^{l} r_{k} \geq \Lambda_{n+1}\right\} & \text { if } q_{n}^{B(p)}=0 .\end{cases}
$$

whereas under the service policy $L(m)$, we have

$$
r_{n+1}^{L(m)}= \begin{cases}r_{n}^{L(m)} & \text { if } s_{n}^{L(m)}<m \text { and } l_{n} L_{n}(m)>0 \\ r_{n}^{L(m)}+1 & \text { if } s_{n}^{l(m)}=m \text { and } q_{n} l(m)>0 \\ \min \left\{l>r_{n}^{L(m)}: \sum_{j=1}^{n} \sigma_{j}+\sum_{k=1}^{L} r_{k} \geq A_{n+1}\right\} & \text { if } q_{n}^{L(m)}=0 .\end{cases}
$$

Notice that both the limited and Bernoulli policiess satisfy $(i=B(p), L(m))$

$$
r_{n+1}^{i}=\min \left\{l>r_{n}^{i}: \sum_{j=1}^{n} \sigma_{j}+\sum_{k=1}^{l} r_{k} \geq \Lambda_{n+1}\right\} \quad \text { if } \quad \varphi_{n}^{i}=0 .
$$

We now introduce the notion of convex increasing ordering $[\overline{7}, 1,270]$. 
(D) We say that a real-valued random variable $X$ is smaller than another real-valued random variable $Y$ in the convex increasing ordering, denoted by $X \leq_{\mathrm{icx}} Y$, if

$$
E[f(X)] \leq E[f(Y)]
$$

for every monotone non-decreasing and convex function $f: \mathbb{R} \rightarrow \mathbb{R}$ for which the expectations are well defined.

We shall make use of the following facts whose proofs can be found in Ross [7, Chap. 8] and Stoyan [9].

(F1) If $X$ and $Y$ are nonnegative random variables, then $X \leq_{\mathrm{icx}} Y$ if and only if

$$
E\left[(X-a)^{+}\right] \leq E\left[(Y-a)^{+}\right], \quad a \geq 0,
$$

with the notation $(x)^{+}=\max \{0, x\}$.

(F2) If $E[X]$ is finite, then it follows from Jensen's inequality that

$$
E[X] \leq_{\mathrm{icx}} X
$$

(F3) Let $Z$ be independent of $X$ and $Y$. If $X \leq_{\mathrm{icx}} Y$, then

$$
X+Z \leq_{\mathrm{icx}} Y+Z \text {. }
$$

\section{The Comparison in The Transient Regime}

In this section, we prove that for suitably chosen parameters $p$ and $m$, the waiting time of the $n$th customer under the policy $L(m)$ is smaller than that under the policy $B(p)$ in the icx ordering. In the next section, this comparison is shown to extend to the steady-state waiting time distributions, whenever they exist. We first prove the following lemmas.

Lemma 4.1 Consider any tu'o service policies $S_{1}$ and $S_{2}$ each satisfying (4). Then, for all $n=1,2, \ldots$ for which $q_{n}^{2}=0$, the inequality

$$
r_{n+1}^{2} \leq r_{n+1}^{1}
$$

holds.

Proof: We obscrve from $(1)$ and $q_{n}^{2}=0$ that

$$
D_{n}^{2}=\sum_{j=1}^{n} \sigma_{\jmath}+\sum_{k=1}^{r_{n}^{2}} r_{k}<A_{n+1}
$$

We consider the following three cases which exhaust all the possibilities.

Case (i) $r_{n}^{1} \leq r_{n}^{2}$ :

By (1), we have $D_{n}^{1} \leq D_{n}^{2}<A_{n+1}$ and so necessarily $q_{n}^{1}=0$. Since $S_{1}$ satisfies (-1). we then have

$$
r_{n+1}^{1}=\min \left\{l>r_{n}^{1}: \sum_{j=1}^{n} \sigma_{j}+\sum_{k=1}^{l} V_{k} \geq \Lambda_{n+1}\right\}
$$


But, because of $(5)$, we can restrict the minimization above to those $l$ for which $l>r_{n}^{2}$, i.e.,

$$
r_{n+1}^{1}=\min \left\{l>r_{n}^{2}: \sum_{j=1}^{n} \sigma_{j}+\sum_{k=1}^{l} V_{k} \geq A_{n+1}\right\},
$$

and therefore $r_{n+1}^{1}=r_{n+1}^{2}$ by making use of (4).

Case (ii) $r_{n}^{1}>r_{n}^{2}$ and $q_{n}^{1}=0$ :

This case is similar to Case (i) since both $q_{n}^{1}$ and $q_{n}^{2}$ are zero. The same argument holds with $S_{1}$ and $S_{2}$ interchanged to yield $r_{n+1}^{1}=r_{n+1}^{2}$.

Case (iii) $r_{n}^{1}>r_{n}^{2}$ and $q_{n}^{1}>0$ :

Since $q_{n}^{1}>0$, we have

$$
D_{n}^{1}=\sum_{j=1}^{n} \sigma_{j}+\sum_{k=1}^{r_{n}^{1}} V_{k}>A_{n+1} .
$$

From the definition of $r_{n+1}^{2}$ in (4), it then follows that

$$
r_{n+1}^{2} \leq r_{n}^{1}
$$

and this last fact trivially implies $r_{n+1}^{2} \leq r_{n+1}^{1}$.

This completes the proof of Lemma 4.1.

Lemma 4.2 Consider a vacation model under the policies $B(p)$ and $L(m)$ where $p$ and $m$ are related by

$$
1-p=1 / m \text {. }
$$

Then, conditioned on a realization of the random sequence $\left\{\tau_{n}, \sigma_{n}, V_{n}, n=1,2, \ldots\right\}$, the comparison

$$
r_{n}^{L(m)} \leq_{\mathrm{icx}} r_{n}^{B(p)} \quad n=1,2, \ldots
$$

holds.

Proof: The proof proceeds by induction. Since the system under both policies satisfies the initial condition stated in assumption (A), we have $r_{1}^{B(p)}=r_{1}^{L(m)}$, and so $(\tau)$ is automatically satisfied for $n=1$. We assume that $(\tau)$ holds for $n=1.2, \ldots, k$ and show that

$$
r_{k+1}^{L(m)} \leq_{\mathrm{icx}} r_{k+1}^{H(p)}
$$

holds.

- We consider three mutually exchusive cases: (i) $q_{h}^{L(m)}>0.1 \leq s_{k}^{L(m)}<m:$ (ii) $q_{k}^{L(m)}>0$. $s_{k}^{L(m)}=m$ : and (iii) $q_{i}^{L(m)}=0$ according to the realization of the system under the policy $L(m)$. Since $L(m)$ is a deterministic policy, given a sample path of $\left\{\tau_{n}, \sigma_{n}, I_{n}, n=1,2 \ldots\right\}$. the realization of the system under this policy is also deterministic. So. One of the three cases above occurs with probability one and the other two with probability zero. Therefure. We can use the induction hypothesis in each case.

We first observe from (2) that for a Bernoulli policy.

$$
r_{n}^{B(n)}+x_{n} \leq r_{n+1}^{R(n)} \quad n=1,2, \ldots
$$


Here, we have used the fact that if $q_{n}^{B(p)}=0$ then the server must take at least one vacation, i.e., $r_{n}^{B(p)}+1 \leq r_{n+1}^{B(p)}$; otherwise, $r_{n+1}^{B(p)}=r_{n}^{B(p)}+x_{n}$.

Case (i) $q_{k}^{L(m)}>0,1 \leq s_{k}^{L(m)}<m$ :

In this case, we have $r_{k+1}^{L(m)}=r_{k}^{L(m)}$ from (3). It then follows from the induction hypothesis and (9) that

$$
r_{k+1}^{L(m)}=r_{k}^{L(m)} \leq_{\mathrm{icx}} r_{k}^{B(p)} \leq_{\mathrm{icx}} r_{k+1}^{B(p)} .
$$

Case (ii) $q_{k}^{L(m)}>0, s_{k}^{L(m)}=m$ :

Since necessarily $k \geq m$, we can apply (9) $m$ times to obtain

$$
r_{k+1-m}^{B(p)}+\sum_{j=k+1-m}^{k} x_{j} \leq r_{k+1}^{B(p)} .
$$

The enforced relation (6) implies that $E\left[\sum_{j=k+1-m}^{k} x_{j}\right]=1$, and so by (F2) we see that

$$
1 \leq_{\mathrm{icx}} \sum_{j=k+1-m}^{k} x_{j} .
$$

Since $r_{k+1-m}^{B(p)}$ is independent of $\left\{x_{j}: j=k+1-m, \ldots, k\right\}$, we conclude from (F3), (10) and the induction hypothesis that

$$
r_{k+1-m}^{L(m)}+1 \leq_{\mathrm{icx}} r_{k+1}^{B(p)} \text {. }
$$

The desired inequality (8) then follows since here we have $r_{k+1-m}^{L(m)}+1=r_{k+1}^{L(m)}$ from (3).

Case (iii) $q_{k}^{L(m)}=0$ :

In this case $r_{k+1}^{L(m)} \leq r_{k+1}^{B(p)}$ by Lemma 4.1 which readily implies (8).

We are now in a position to prove the main result of this section.

Theorem 4.1 Consider a vacation model with deterministic vacation periods, i.e.,

$$
V_{n}=V, \quad n=1,2, \ldots
$$

for some constant $V$. For policies $B(p)$ and $L(m)$ satisfying (6). the comparison

$$
\mathrm{W}_{n}^{+L(m)} \leq_{\mathrm{icx}} W_{n}^{B(p)} \quad n=1,2, \ldots
$$

holds.

Proof: From (1) and (11), we conclude that for $i=B(p), L(m)$

$$
D_{n}^{i}=\sum_{j=1}^{n} \sigma_{j}+V r^{i}{ }^{i}, \quad n=1.2 \ldots .
$$

Let us consider a sample path of $\left\{\tau_{n}, \sigma_{n}, n=1,2, \ldots\right\}$. We see from Lemma 4.2 and (F.3) that. conditioned on this sample path, we have $D_{n}^{L(m)} \leq_{i c x} D_{n}^{B(p)}$. Since the waiting time of $C_{n}$ can be expressed as

$$
H_{n}^{i}=D_{n}^{i}-\sigma_{n}-A_{n} \quad i=B(l), L(m): n=1,2 \ldots,
$$

we again obtain from $(F, 3)$ that $W_{n}^{L(m)} \leq_{\mathrm{i} \times \mathrm{x}} W_{n}^{B(p)}$. Unconditioning with respect to $\left\{\tau_{n}, \sigma_{n}, n=\right.$ $1,2, \ldots\}$ completes the prod. 


\section{The Comparison in The Steady-State Regime}

In this section, the comparison result derived in the previous section is extended to the steadystate regime. We assume that under both $B(p)$ and $L(m)$ (with $p$ and $m$ related by (6)) the vacation model is stable, i.e.,

$$
W_{n}^{B(p)} \stackrel{D}{\longrightarrow} W^{B(p)} \quad \text { and } \quad W_{n}^{L(m)} \stackrel{D}{\longrightarrow} W^{L(m)},
$$

where $W^{B(p)}$ and $W^{L(m)}$ are almost surely finite and $\stackrel{D}{\longrightarrow}$ denotes convergence in distribution. It is well known $[5,6]$ that the necessary and sufficient condition for this stability is given by

$$
\bar{\sigma}+(1-p) V=\bar{\sigma}+\frac{1}{m} V<\bar{\tau}
$$

where $\bar{\sigma}=E\left[\sigma_{1}\right]$ and $\bar{\tau}=E\left[\tau_{1}\right]$.

Theorem 5.1 For the vacation model of Theorem 4.1 under the stability condition (13), the comparison

$$
W^{L(m)} \leq_{\mathrm{icx}} W^{B(p)}
$$

holds.

Before we prove the theorem above, we first establish some general results stated in the following two lemmas.

Lemma 5.1 Let a modified vacation model $\left\{\hat{\tau}_{n}, \hat{\sigma}_{n}, n=1,2, \ldots\right\}$ be constructed from the random variables $\left\{\tau_{n}, \sigma_{n}, x_{n}, n=1,2, \ldots\right\}$ where $\hat{\tau}_{n}$ and $\hat{\sigma}_{n}$ are given by

$$
\hat{\tau}_{n}=\tau_{n} \quad \hat{\sigma}_{n}=\sigma_{n}+x_{n} V, \quad n=1,2, \ldots
$$

and the length of each vacation period is given by $V$. Then, the waiting time $\hat{W}_{n}^{E}$ of the nth customer in this vacation model under the exhaustive service policy is identical to that of the original vacation model under the Bernoulli policy which corresponds to the sequence $\left\{x_{n}, n=1,2, \ldots\right\}$, i.e..

$$
\hat{W}_{n}^{E}=W_{n}^{B(p)}, \quad n=1.2, \ldots
$$

Proof: The proof proceeds by induction. Since in both systems the waiting time of the first customer is simply the remaining vacation when that customer arrives. (16) trivially holds for $n=1$. We take as induction hypothesis that

$$
\hat{\mathbb{H}}_{n}^{E}=W_{n}^{B(n)}=: W_{n}, \quad n=1, \ldots, k
$$

and sliow that

$$
\hat{H}_{k+1}^{-E^{\prime}}=H_{k+1}^{+R(p)}
$$

For the policy $B(y)$, we have

$$
W_{k+1}^{B(p)}= \begin{cases}W_{k}+\sigma_{k}+x_{k} V-\tau_{k+1} & \text { if } W_{k}+\sigma_{k}-\tau_{k+1} \geq 0 \\ W_{k}+\sigma_{k}-\tau_{k+1}+j_{k}^{B V} & \text { otherwise, }\end{cases}
$$


where

$$
j_{k}^{B}=\min \left\{j \geq 0: W_{k}+\sigma_{k}-\tau_{k+1}+j V \geq 0\right\}
$$

From the description of the exhaustive policy and (15), $\hat{W}_{k+1}^{E}$ is given by

$$
\hat{W}_{k+1}^{E}=W_{k}+\sigma_{k}+x_{k} V-\tau_{k+1}+j_{k}^{E} V
$$

where

$$
j_{k}^{E}=\min \left\{j \geq 0: W_{k}+\sigma_{k}+x_{k} V-\tau_{k+1}+j V \geq 0\right\} .
$$

Since $x_{k} \geq 0$, we have $W_{k}+\sigma_{k}+x_{k} V-\tau_{k+1} \geq 0$ whenever $W_{k}+\sigma_{k}-\tau_{k+1} \geq 0$, and so $\hat{W}_{k+1}^{E}$ can be expressed as

$$
\hat{W}_{k+1}^{E}= \begin{cases}W_{k}+\sigma_{k}+x_{k} V-\tau_{k+1} & \text { if } W_{k}+\sigma_{k}-\tau_{k+1} \geq 0 \\ W_{k}+\sigma_{k}-\tau_{k+1}+\left(x_{k}+j_{k}^{E}\right) V & \text { otherwise. }\end{cases}
$$

To obtain (17), it remains only to show that

$$
x_{k}+j_{k}^{E}=j_{k}^{B} .
$$

But, this is apparent from (18) and (20) upon examining the cases where $x_{k}=0$ and $x_{k}=1$ separately.

Lemma 5.2 Let $\left\{X_{n}, n=1,2, \ldots\right\}$ and $X$ be nonnegative random variables and suppose that $X_{n} \stackrel{D}{\longrightarrow} X$. If there exists an integrable nonnegative random variable $Y$ such that

$$
X_{n} \leq_{\mathrm{icx}} Y, \quad n=1,2, \ldots,
$$

then $\mathrm{X}$ is also integrable and moreover

$$
\lim _{n \rightarrow \infty} E\left[X_{n}\right]=E[X]
$$

Proof: Taking $f(x)=x$ in the definition of icx ordering, we obtain from (21) that

$$
E\left[X_{n}\right] \leq E[Y], \quad n=1,2, \ldots
$$

Since $X_{n} \stackrel{D}{\longrightarrow} X$, it follows by $[1$, Theorem 5.3] that

$$
E[X] \leq \lim _{n} \inf E\left[X_{n}\right]
$$

Combining these inequalities yields

$$
E[X] \leq E\left[Y^{\circ}\right]<\infty
$$

and so $X$ is integrable. For each $a \geq 0$, defne a mapping $f_{n}: \mathbb{R} R_{+} \rightarrow \mathbb{R} R_{+}$by

$$
f_{a}= \begin{cases}x & \text { if } 0 \leq x<a \\ a & \text { if } x \geq a\end{cases}
$$

Since $f_{a}$ is bounded and continuous, part (iii) of [1, Theorem 5.2] thus yichds

$$
\lim _{n \rightarrow \infty} I:\left[f_{n}\left(X_{n}\right)\right]=E\left[f_{n}(X)\right]
$$


Combining this fact with

$$
E\left[X_{n}\right]=E\left[f_{a}\left(X_{n}\right)\right]+E\left[\left(X_{n}-a\right)^{+}\right]
$$

and

$$
E[X]=E\left[f_{a}(X)\right]+E\left[(X-a)^{+}\right]
$$

we obtain

$$
\limsup _{n}\left|E\left[X_{n}\right]-E[X]\right| \leq \sup _{n} E\left[\left(X_{n}-a\right)^{+}\right]+E\left[(X-a)^{+}\right] .
$$

But, by (F1), we have from (21) that

$$
E\left[\left(X_{n}-a\right)^{+}\right] \leq E\left[(Y-a)^{+}\right], \quad n=1,2, \ldots,
$$

and so

$$
\limsup _{n}\left|E\left[X_{n}\right]-E[X]\right| \leq E\left[(Y-a)^{+}\right]+E\left[(X-a)^{+}\right] .
$$

The proof of (22) is now completed by letting $a \rightarrow \infty$ and using the fact that both $X$ and $Y$ are integrable.

Proof of Theorem 5.1: Only the case where $E\left[W^{B(p)}\right]<\infty$ needs to be considered since $E\left[W^{B(p)}\right]=\infty$ trivially implies (14). By Proposition 1.3 .2 of [9], (14) directly follows if we can show that the limits

$$
\left.\left.\lim _{n \rightarrow \infty} E\left[W_{n}^{B(p)}\right)\right]=E\left[W^{B(p)}\right] \text { and } \lim _{n \rightarrow \infty} E\left[W_{n}^{L(m)}\right)\right]=E\left[W^{L(m)}\right]
$$

hold true and finite. To that end, we shall make use of Lemmas 5.1 and 5.2.

Doshi [2] established a sample path comparison between a vacation model under the exhaustive policy and its corresponding $G I / G I / 1$ queue (the same system with no vacations). Using this result, $\hat{W}_{n}^{E}$ can be decomposed into the waiting time $\hat{W}_{n}$ of the $n$th customer in the corresponding $G I / G I / 1$ queue and a term $Y_{n}$ that corresponds to the vacations. The term $Y_{n}$ is always smaller than the last vacation period taken, and so

$$
\hat{W}_{n}^{E} \leq \hat{W}_{n}+V, \quad n=1,2, \ldots
$$

Note that under the stability assumption, the corresponding $G I / G I / 1$ queue is also stable, i.e.. $\hat{W}_{n} \stackrel{D}{\longrightarrow} \hat{W}$ for some nondefective $\hat{W}$. Moreover, since $E\left[W^{B(p)}\right]$ is finite, so is $E\left[\hat{W}^{\gamma}\right]$. Theorem 5.1 .1 of [9] thus yields

$$
\hat{W}_{n} \leq_{\mathrm{icx}} \hat{H} \cdot \quad n=1.2 \ldots
$$

This inequality, together with (16) and (2.1) implies that

$$
\mathbb{H}_{n}^{B(p)} \leq_{\mathrm{icx}} \hat{H}+\boldsymbol{V}, \quad n=1,2 \ldots
$$

Subsequently, a use of Theorem 4.1 yields

$$
W_{n}^{L(m)} \leq_{\mathrm{i} \times \mathrm{x}} \mathbb{H}+\mathrm{r}, \quad n=1,2, \ldots
$$

By Lemma 5.2. the icx-ortering bounds (25) and (26), together with (13) radily imply (23). and so the proof of theorem 5.1 is complete. 


\section{References}

[1] P. Billingsley, Convergence of Probability Measures, John Wiley \& Sons, New York, 1968.

[2] B.T. Doshi, "A Note on Stochastic Decomposition in A GI/G/1 Queue with Vacations or Set-Up Times," J. Appl. Prob. 22, 1985, pp. 419-428.

[3] B.T. Doshi, "Queueing Systems with Vacations-A Survey," Queueing Systems 1, 1986, pp. 29-66.

[4] S.W. Fuhrmann, "Inequalities for Cyclic Service Systems with Limited Service Disciplines," Proc. Globecom '87, 1987, pp. 182-186.

[5] J. Keilson and L.D. Servi, "Oscillating Random Walk Models for GI/G/1 Vacation Systems with Bernoulli Schedules," J. Appl. Prob. 23, 1986, pp. 790-802.

[6] P.J. Kuehn, "Multiqueue Systems with Nonexhaustive Cyclic Service," The Bell System Tech. J. 58 (3), 1979, pp. 671-698.

[7] S. Ross, Stochastic Processes, John Wiley \& Sons, New York, 1983.

[8] L.D. Servi and D.D. Yao, "Stochastic Bounds for Queueing Systems with Limited Service Schedules," Performance Evaluation 9, 1989, pp. 247-261.

[9] D. Stoyan, Comparison Methods for Queues and Other Stochastic Models (English translation, ed. D. J. Daley), John Wiley \& Sons, New York, 1984. 